
Ginter Dem Sađregifter befindet jid ein ausfübrlides Werzeidnis Der

\section{Guttentaginen Sammlung}

\section{Deutjaer Reid) $=$ und $\mathfrak{P r e u} \tilde{B}$ ijđer Gejeze}

- Tertausgaben mit Anmerkungen; Tajoenformat -

bie alle widtigeren Gejebe in unbebingt $z$ uver=

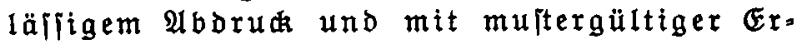
läuterung wiedergibt. 
Guttentagi 中e $S_{\mathfrak{a}} \mathfrak{m} \mathfrak{m} \mathfrak{u} \mathfrak{g}$

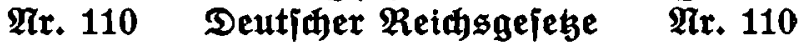

Jertausgaben mit Anmerkungen und Sadjegititer

\section{Gejeg \\ über einen einmaligen auker $=$ ordentlichen Webrbeitrag}

nebit ben $\mathfrak{A}$ usführungsbeitimmungen bes $\mathfrak{B u n b e s}=$ rats und den preuß̉iłden $\mathfrak{A}$ usführungsworidriften

Textausgabe mit Anmerkungen und Sadregiiter

von

A. Fernom

Geheimer Oberfinanzrat und vorttagenber Rat

im Finarzminifterium

$\underline{\text { 3weite, vermehrte und verbefferte } \mathfrak{A} \text { uflage }}$

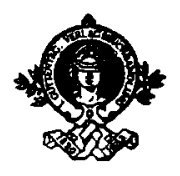

Berlin 1914.

3. Guttentag, $\mathfrak{V e r l a g s b u d h a n d i u n g , ~}$

F. $\mathfrak{m} . \mathfrak{b} . \mathfrak{G}$. 


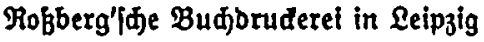

